\title{
Calculating the Band Gaps of Perfect Carbon Nanotube through Tight Binding Method
}

\author{
Rashid Nizam ${ }^{1}$, Mirza Mohd Sehban ${ }^{2}$ \\ ${ }^{1}$ Department of Physics, IFTM University, Moradabad, India \\ ${ }^{2}$ Department of B.Ed. Rashida Begum Muslim Degree College, Amroha, India
}

\begin{abstract}
Eighty three types perfect carbon nanotubes (PCNTs) are chosen between the diameter range of $0.3 \mathrm{~nm}-3.9355 \mathrm{~nm}$. These PCNTs include armchair, chiral and zigzag tubes leaving out those zigzag tubes which are metallic in nature. 16 of these 83 PCNTs are metallic (armchair) and 67 are semiconductor (chiral and zigzag). The band gaps of chiral and zigzag tubes are calculated with increasing chiral angle $\theta^{\circ}$ from $0.3 \mathrm{~nm}$ to $3.9355 \mathrm{~nm}$ diameters (67 in numbers) in order of increase in indices (n, m). The tube diameter and the calculated value of band gap in also given. It is observed that irrespective of the chiral angle, with the increase in diameter the band gap decreases. Further it may be seen that for the tube of about the same diameter with different chirality shall have about the same band gap. Therefore it is seen that the band gap of the semiconducting tubes depend inversely upon the diameter. Also calculated that the armchair $(n, n)$ tubes band gap of the order of $0.011 \mathrm{eV}$ remain independent of diameter.
\end{abstract}

Keywords: Tight Binding Method, carbon nanotube, DOS

\section{Introduction}

The electronic band structure (or simply band structure) of a solid is defined as those ranges of energy an electron is "forbidden" or "allowed" to possess and is equivalent to the energy required to free an outer shell electron from its orbit about the nucleus to become mobile charge carrier and able to move freely within the solid material $[2,4]$. It gives a band gap or energy gap within the energy range in a solid where no electron states can exist and is an important factor for determination of electrical conductivity of materials. The band structure for a periodic specific crystal lattice may be obtained from diffraction of quantum mechanical electron waves along the Bravais lattice. Material properties such as electronic, optical and others depend upon band structure [2]. In the present work numerical calculations have been presented for energy gaps for various perfect carbon nanotubes (PCNTs) and electron density of states (DOS) in Sub-Sections respectively.

Directions of each wave vector and no net charge is transported in either direction In the semi-classical theory (Tight Binding Method) the time evolution of the wave vector for the Bloch electrons in a uniform electric field electron interchange of states occur through each carbon nanotube [6]. Each of these carbon nanotubes exhibits its own electronic band and the band structure changes when electric or magnetic field is applied. The band structure of these nanotubes describe whether it is conducting or semiconducting. This motivates the intensive study of the electronic band structure of nanotubes since their shape and occupation are crucial for the electronic transport. In the present chapter, the band structure and the band gaps of various PCNTs are studied. This section gives formation of different forms of carbon nanotubes using two dimensional graphene sheets, namely: the armchair; the zigzag; and the chiral types of the carbon nanotubes. Electrons Density of State for these CNTs is calculated using the Tight Binding Method. Finally, the energy gaps are calculated for each type of carbon nanotubes.

\section{Computational Details}

\subsection{Model}

\subsection{Types of Perfect Carbon Nanotubes (PCNTs) Studied}

The construction of perfect carbon nanotube has been explained. Using the Tight Binding Method [5, 6] eightythree carbon nanotubes is constructed [1-3] and out of these four tubes are shown in Figure 1- Figure 4, two are zigzag and the other two have armchair structures.

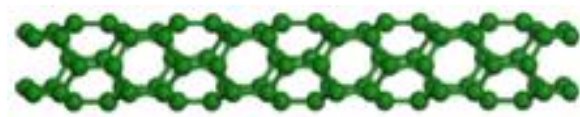

Figure 1: Perfect carbon nanotube (zigzag $(4,0)$ )

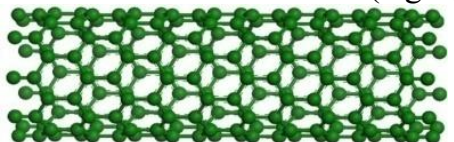

Figure 2: Perfect carbon nanotube (zigzag $(9,0)$ )

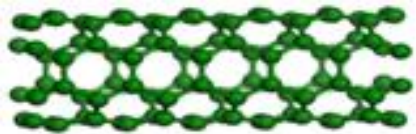

Figure 3: Perfect carbon nanotube (armchair $(4,4))$

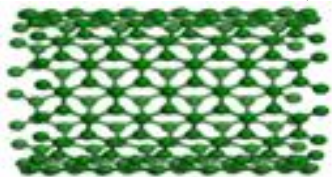

Figure 4: Perfect carbon nanotube (armchair $(9,9))$

\section{Calculations of Energy Gap}

\subsection{Tight Binding Method}

Tight Binding Method (TBM) or LCAO (linear combination of atomic orbitals) or Bloch is the standard method for solving the periodic potential problems encountered in the electronic motion in solids. This was originally proposed by 


\section{International Journal of Science and Research (IJSR) \\ ISSN (Online): 2319-7064}

Index Copernicus Value (2015): 78.96 | Impact Factor (2015): 6.391

Bloch. The method consists of making a linear combination of atomic orbitals situated on the various atoms of the crystal. The coefficients being the values of the plane wave $\exp (\mathrm{ik} . \mathrm{R})$ at various positions $\mathrm{R}$ where the atoms are located.

TBM gives solutions exhibiting all the correct symmetry properties of the energy bands. It is easier to get solutions with TBM for energy bands at arbitrary points in the Brillouin zone in comparison with other approximate methods. Initially an atomic orbital $\varphi_{n}\left(r-R_{i}\right)$ is located on an atom at vector position $R_{i}$ and with quantum numbers symbolized by the subscript $n$. Then the Bloch sum is formulated over all the atomic positions in the unit cells of the crystal $\sum R_{i} \exp (i k . R) \varphi_{n}(r-R i)$. Similarly, other Bloch sums are set up corresponding to each atomic orbital of an atom which corresponds to each atom in the unit cell of the crystal. The non-vanishing matrix components of the Hamiltonian lies between the Bloch sums of the same $\mathrm{k}$ value corresponding either to different atomic orbitals on the same atom or to atomic orbitals on different atoms in the unit cell. Diagonalizing the matrix of Bloch sum yields the energy band structure solution. Non-diagonal matrix components vanish at special symmetry points of the Brillouin zone by taking special values of $\mathrm{k}$. These vanishing components of matrix lead to special properties of the energy bands at these symmetry points.

The commonly used method for determining the electronic structure of single wall carbon nanotubes is the Tight Binding (TBM) Method [6]. This is a standard procedure in solid-state physics and works well for the case of weak overlap of atomic wave functions in an insulating crystal. The TBM approximation gives a convenient method for understanding the basic characteristics of band structure in single wall carbon nanotube $[5,6]$. The electronic structure of a carbon nanotube (CNT) can be acquired from that of graphene. The wave vector related with the chiral vector $C_{h}$ in the circumferential direction gets quantized. On the other hand wave-vector associated with the direction of the translation vector $\mathrm{T}$ along the CNT axis remains continuous for an infinite carbon nanotube. These are the boundary conditions of the nanotube. This gives the energy bands in form of one-dimensional dispersion relationship which are the cross sections of the energy bands in the case of graphene. For the reciprocal lattice vectors $K_{2}$ of PCNTs along the carbon nanotube axis $C_{h} . K_{2}=0, T \cdot K_{2}=2 \pi$ and $K_{1}$ in the circumferential direction, $\left(C_{h} . K_{1}=2 \pi, T . K_{1}=\right.$ $0)$ the expressions are given as:

$$
\begin{gathered}
K_{1}=\frac{1}{N}\left(-t_{2} b_{1}+t_{1} b_{2}\right) \\
K_{2}=\frac{1}{N}\left(m b_{1}-n b_{2}\right)
\end{gathered}
$$

The one-dimensional energy dispersion relation of a carbon nanotube can be written as

$$
E_{C N T}^{v}(k)=E_{g-2 D}\left(k \frac{K_{2}}{\left|K_{2}\right|}+v K_{1}\right)
$$

where $-\pi / T<k<\pi / T$ is a one-dimensional wavevector along the carbon nanotube axis and $v=1,2,3 \ldots \ldots \ldots N$. The periodic boundary condition for carbon nanotube provides $N$ discrete $k$ values in the circumferential direction. These $N$ pairs of energy dispersion curves correspond to the cross sections of the two- dimensional energy dispersion surface of graphene which is given by eqn. (3). Several lines cut near one of the $K$ points as shown in Figure 5. The separations between two adjacent lines with the length of the cutting lines are given by $\left|K_{1}\right|=2 / d_{C N T}$ and $\left|K_{2}\right|=2 \pi / T$ respectively. The carbon nanotube gets a zero energy gap if the cutting line passes through a $K$ point of the two-dimensional Brillouin zone [2, 7] as shown in Figure 5(a), where the $\pi$ and $\pi^{*}$ energy bands of graphene are degenerate by symmetry. If the $K$ point is situated between two cutting lines such that $K$ is always located in a position one-third of the distance between two adjacent $K_{1}$ lines then a semiconducting carbon nanotube with a finite energy gap is formed as shown in Figure 5 (b). Further, for a given indices of carbon nanotube $(n, m)$ if $n-m$ is exactly divisible by 3 then the carbon nanotube is metallic where as carbon nanotubes with residuals 1 and 2 for the division $n-m$ by 3 are semiconducting as pictorially depicted in Figure 5 (a) and Figure 5 (b) respectively. Calculations for band gaps of different kind of carbon nanotubes are given below that explain the metallic or semiconductor nature of the carbon nanotube.

(a) Metallic

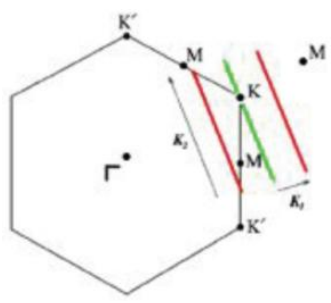

(b) Semiconductor

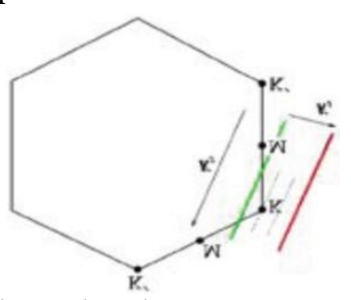

Figure 5: One-dimensional wave-vectors $\mathrm{K}$ are shown in the BRILLOUIN zone of graphene as bold lines for (a) metallic and (b) semiconducting carbon nanotubes [ref. 2, 7]

\section{Result of armchair and zigzag PCNTs}

When the system has a large number of atoms in case of PCNT or CNT with Intramolecular Junction as also in crystalline material, the higher energy levels tend to merge into two separate bands of allowed energies called the valence band and the conduction band as shown in Figure 6 and Figure 7. The energy levels which are mostly filled are called valence band while those which remain nearly empty are called conduction band. Electrons that occupy the energy levels in the conduction band are called free elections. The extremely closely spaced energy levels in the valence and conduction bands are often separated by an energy range where there are no allowed quantum states or energy levels known as the energy gap $E_{g}$ (or band gap).

Fermi energy level at $E_{F}=0$ divides the conduction band and valence band. It is useful to build an approximate relation that describes the dispersion relations in the regions 


\section{International Journal of Science and Research (IJSR) \\ ISSN (Online): 2319-7064 \\ Index Copernicus Value (2015): 78.96 | Impact Factor (2015): 6.391}

around the Fermi energy $E_{F}=0$ as electrical conduction is decided by states around the Fermi energy. The expression around the point $(0, \pm 4 \pi / 3 a)$ where the energy gap is zero and $f=0$ is given by $f(k) \approx-t\left(1+2 e^{\sqrt{3} k_{x} a / 2} \cos \left(k_{y} a / 2\right)\right)$. It is easy to show that $f(k) \approx(i \sqrt{3} a t / 2)\left(k_{x} \mp i \beta_{y}\right)$, with $\beta_{y} \equiv k_{y} \mp$ $(4 \pi / 3 a)$. The corresponding energy dispersion relation can be written as [28]

$$
\begin{gathered}
E_{g-2 D}(k) \quad|f(k)| \\
E_{g-2 D}(k) \frac{\sqrt{3} a t}{2} \sqrt{k_{x}^{2}+\beta_{y}^{2}}
\end{gathered}
$$

\subsection{Band Structure}

In general $(n, n)$ armchair carbon nanotubes yield $4 n$ energy subbands by means of $2 n$ conduction and $2 n$ valence bands. Out of these $2 n$ bands, two are non-degenerate and n-1are doubly degenerate [2]. The degeneracy comes from the two subbands with the same energy dispersion but with different $v$ - values. In general all the zigzag carbon nanotubes have the lowest conduction and the highest valence bands and are doubly degenerate whereas all armchair carbon nanotubes have band degeneracy between the highest valence and the lowest conduction band. For both of the armchair as well as zigzag carbon nanotubes bands are symmetric with respect to $\mathrm{k}=0[2-4]$.
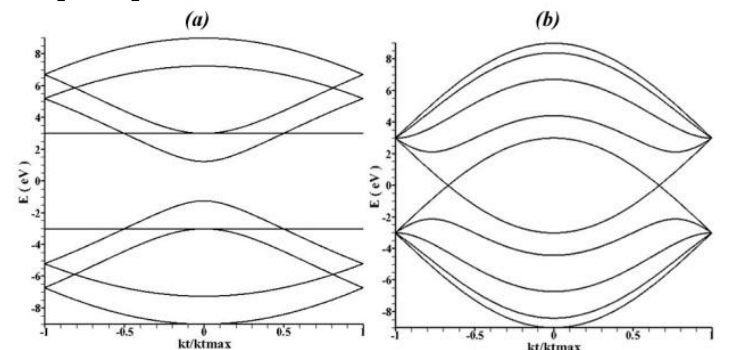

Figure 6: Energy versus Axial Wave Vector (a) of zigzag, semiconducting $(4,0)$ and (b) of armchair, metallic $(4,4)$ PCNTs respectively
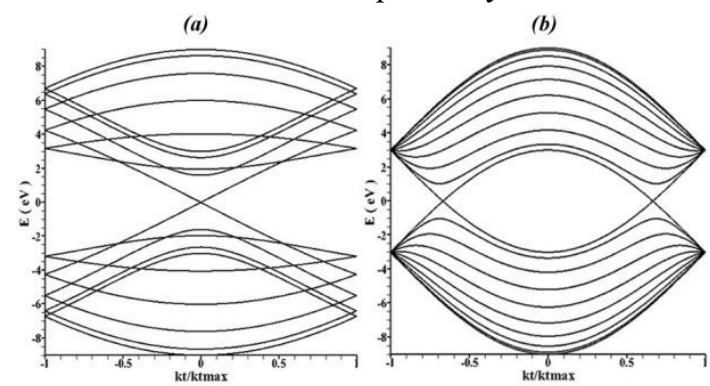

Figure 7: Energy versus Axial Wave Vector (a) of zigzag, metallic $(9,0)$ and (b) of armchair, metallic $(9,9)$ PCNTs respectively

It is a known fact [2] and present calculations also show that an armchair carbon nanotubes bands have two valleys at around $\mathrm{k}= \pm 2 \pi / 3 \mathrm{a}$ points as shown for example in Figure 7(b) while the zigzag carbon nanotubes can have at the most one valley as shown in Figure 7(a). The bands in each armchair cross the Fermi level at $\mathrm{k}= \pm 2 \pi / 3 \mathrm{a}$ thus they are considered to exhibit metallic behaviour [2]. There is no energy gap of the order of $\approx 0.011 \mathrm{eV}$ for armchairs as shown in Figure 6(b), Figure $7(b)$ and Figure 8 irrespectively of the tube diameter. Some zigzag tubes also show zero band gaps as shown in Figure 7(a). This is because zigzag tubes become metallic when the tubes are $(2 n+m) / 3$ is an integer otherwise the tube remains semiconducting.

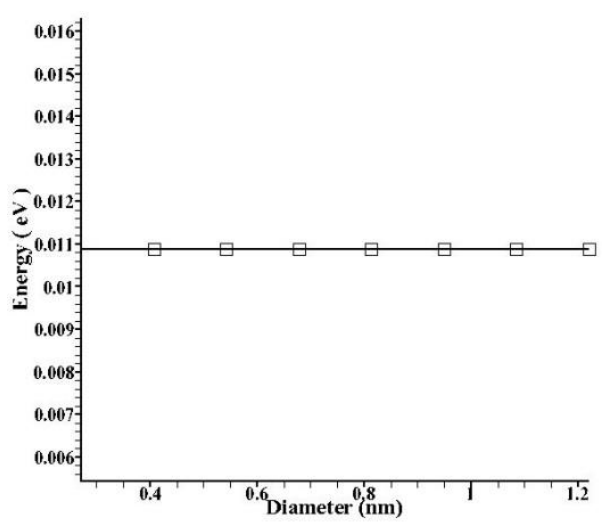

Figure 8: Calculated band gaps in PCNTs (armchair) of different diameters.

83 PCNTs are chosen between the diameter range of $0.3 \mathrm{~nm}$ - $3.9355 \mathrm{~nm}$. These PCNTs include armchair, chiral and zigzag tubes leaving out those zigzag tubes which are metallic in nature. 16 of these 83 PCNTs are metallic (armchair) and 67 are semiconductor (chiral and zigzag). The band gaps of chiral and zigzag tubes between $0.3 \mathrm{~nm}$ to $3.9355 \mathrm{~nm}$ diameters (67 in numbers) is presented in Figure 9 and in Table 1.

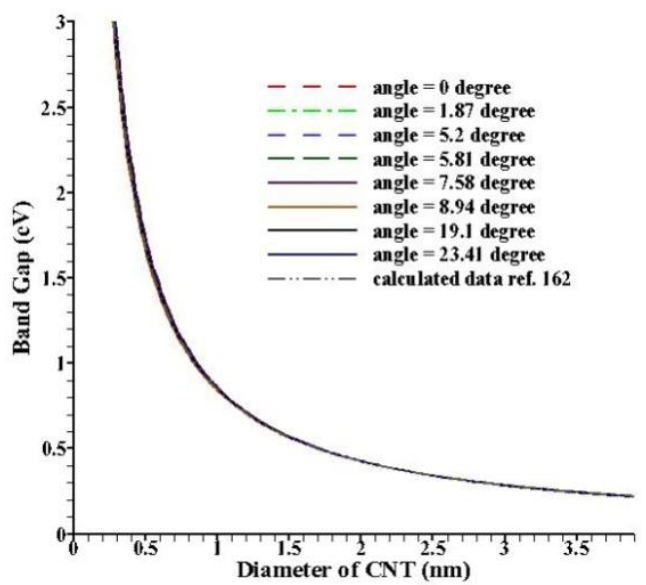

Figure 9: Band gaps of PCNTs with increase in diameters.

In Tables 1 nanotubes are listed with increasing chiral angle $\theta^{\circ}$ in order of increase in indices $(n, m)$. The tube diameter and the calculated value of band gap in also given. The results in Tables 1 clearly show that irrespective of the chiral angle, with the increase in diameter the band gap decreases. Further it may be seen that for the tube of about the same diameter with different chirality shall have about the same band gap. Therefore it is clear that the band gap of the semiconducting tubes depend inversely upon the diameter. The armchair $(n, n)$ tubes band gap of the order of $0.011 \mathrm{eV}$ remain independent of diameter as shown in Figure 9 and Figure 8. Figure 9 also give the band gaps by Mintmire et al [8]. Similar results have also been reported by White et al $[10,11]$ for the band gaps of carbon nanotubes. 


\subsection{Electron Density of States (DOS)}

Density of states (DOS) is the total number of energy levels per unit volume available for possible occupation by electrons. Local density of states (LDOS) is defined as the specific small area where the total number of electrons occupy energy levels per unit volume.

\subsection{DOS for zigzag PCNT}

The energy bands for $(n, 0)$ zigzag carbon nanotubes may be obtained by enforcing the periodic boundary conditions. This prescribes the allowed wave-vectors $\mathrm{k}_{\mathrm{y}}$ in the circumferential direction as $\mathrm{nk}_{\mathrm{y}} \mathrm{a}=2 v \pi,(v=1 \ldots 2 \mathrm{n})$. The one-dimensional dispersion relations for the $4 n$ states of the $(n, 0)$ zigzag carbon nanotubes may be written as:

$$
\begin{gathered}
E^{v}\left(k_{x}\right) \quad \frac{\sqrt{3} a t}{2} \sqrt{k_{x}^{2}+\left[\frac{4 \pi}{3 a}\left(\frac{3 v}{2 n}-1\right)\right]^{2}} \\
-\pi / \sqrt{3} a<k_{x}<\pi / \sqrt{3} a
\end{gathered}
$$

The energy gap for subband $v$ can be written as difference between the energies of the + and - branches at $\mathrm{k}_{\mathrm{x}}=0$ :

$$
E_{g}^{v}=\sqrt{3} a t \frac{2 \pi}{n a}\left(v-\frac{2 n}{3}\right)
$$

The energy gap has a minimum value of zero corresponding to $v=2 \mathrm{n} / 3$. If $\mathrm{n}$ is not a multiple of three then the minimum value of $v-2 n / 3$ is equal to $1 / 3$. This gives the minimum energy gap as:

$$
E_{g}=\frac{\sqrt{3} a t}{3} \frac{2 \pi}{n a}=\frac{2 a_{C-C} t}{d_{C N T}} \approx \frac{0.8 e V_{n m}}{d_{C N T}}
$$

where $d_{C N T}-n a / \pi$ is the diameter of the carbon nanotubes. The DOS for semiconducting zigzag carbon nanotubes based on (6) and (7) may be written as:

$$
g(E)=\sum_{v} \frac{8}{3 \pi a_{C-C} t} \frac{E}{\sqrt{E-E_{g}^{v} / 2}}
$$

which is valid only if $\left(E-E_{F}\right) \ll t[29]$.

The DOS for the metallic carbon nanotubes (armchair) DOS may also be obtained but it is independent of their diameters as well as chirality because of the linear dispersion relations around the Fermi energy [2]. The density of states (DOS) [2] per unit length along the metallic carbon nanotube axis is constant given by $8 / 3 \pi \mathrm{a}_{\mathrm{C}-\mathrm{C}} \mathrm{t}$.

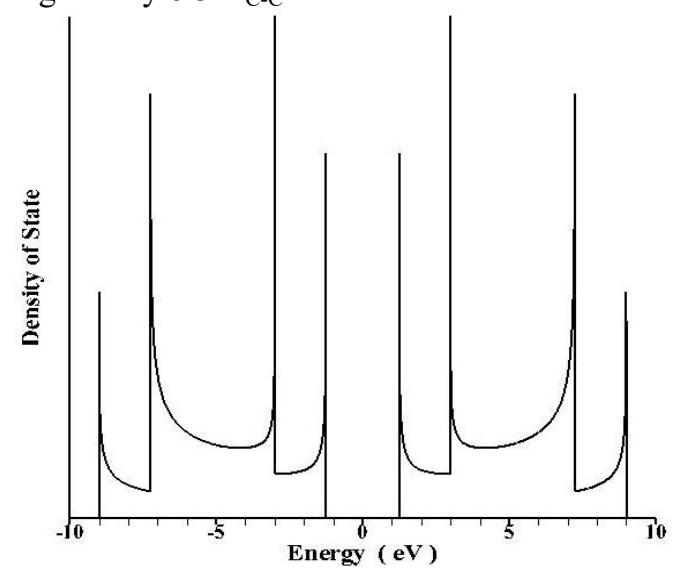

(a)

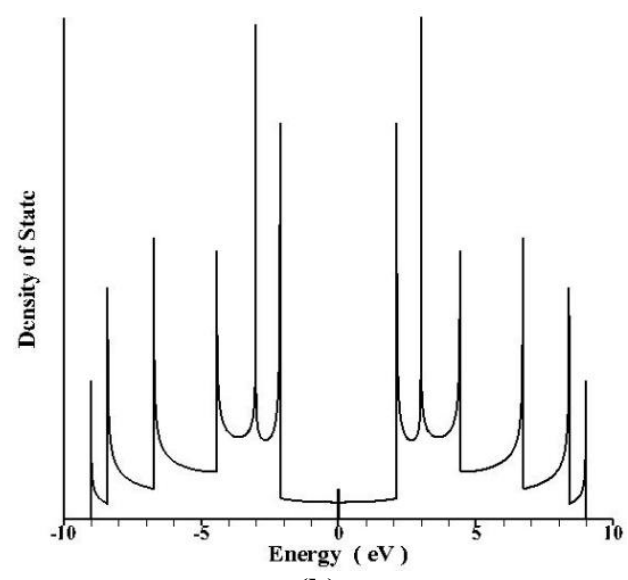

(b)

Figure 10 Density of State versus Energy (a) of $(4,0)$ (zigzag, semiconducting) and (b) of $(4,4)$ (armchair, metallic) PCNTs respectively.

It is observed that the DOS of PCNTs shows same typical features in one dimensional system. The DOS of semiconducting zigzag depends on the structure and diameter as shown in Figure 10 (a) and Figure 11 (a). The continuous electronic density of states (DOS) in a CNT is divided into a series of spikes because of radial confinement of the wave function which is referred to as Van Hove singularities shown in Figure 10 and Figure 11. It may be seen in Figures 10(b) and 11(b) observed that the universal relation for the armchair [2] nanotubes has zero band gaps near the Fermi level.

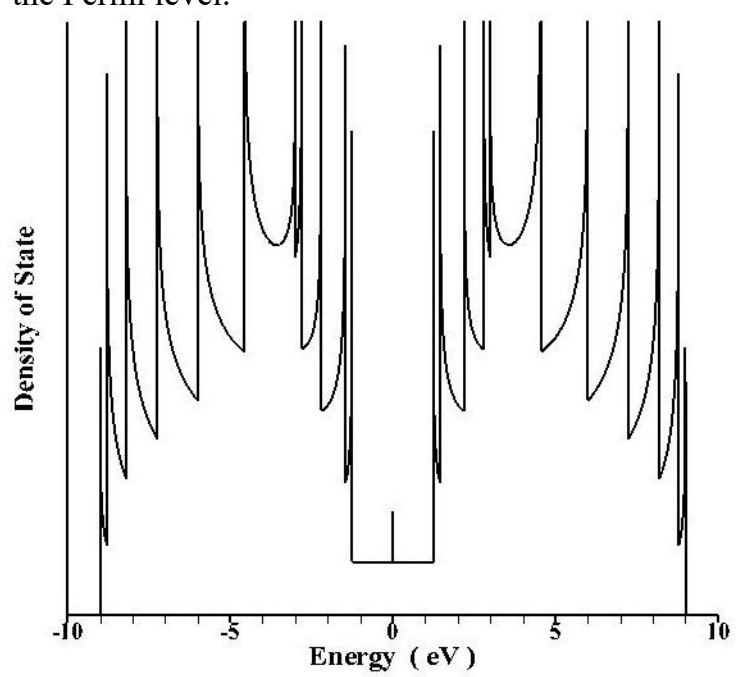

(a) 


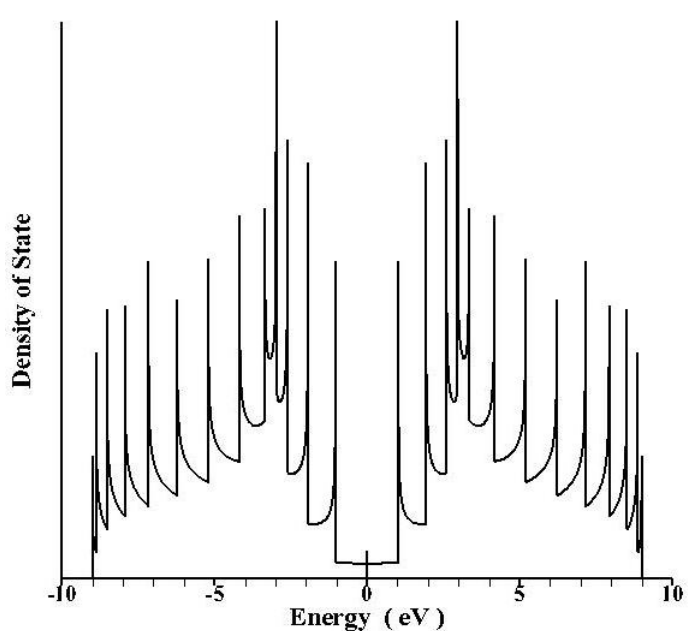

(b)

Figure 11 Density of State versus Energy (a) of $(9,0)$ (zigzag, metallic) and (b) of $(9,9)$ (armchair, metallic) PCNTs respectively

At Fermi level the DOS between the two adjacent Van Hove singularities has some finite value for metallic tubes as shown in Figure 10(b) and Figure 11(b) while the DOS is zero at the Fermi level for semiconducting nature of zigzag tubes shown in Figure 10 (a). It may be noted that the zigzag tube $(9,0)$ is metallic in nature. It has been already verified through Scanning-Tunneling Microscopy (STM) that the nanotubes [9] that both metallic and semiconducting PCNTs possess DOS.

\section{Conclusion}

This paper deals with the electronic band structures of onedimensional carbon nanotubes and the effect of change in diameter of carbon nanotubes on the band structure and explains the behavior of PCNTs in respect of being metallic or semiconductor depending upon the chirality. In case of graphene, a zero gap semi-conductor, the valence and conduction bands meet at the corners called the K-points of the hexagonal Brillouin zone. The graphene sheet can be taken as infinite two dimensional plane without additional constrains while the PCNTs made up of rolling of graphene sheet may be considered to be infinite in one direction and finite around its circumference producing its confinement effects and additional periodic boundary conditions. Eightythree perfect carbon nanotubes are considered for calculations of band gaps. In the Section electron DOS is calculated and presented.

\section{References}

[1] M. P. Anantram and F. Leonard, "Physics of carbon nanotube electronic devices", Rev. Prog. Phys., Vol. 69 p. 507, 2006.

[2] S. Iijima, "Helical microtubules of graphitic carbon", Nature 354, 56, 1991.
[3] R. Saito, G. Dresselhaus and M. S. Dresselhaus, "Physical Properties of Carbon Nanotubes", Imperial College Press, London, 1998.

[4] M. Fujita, R. Saito, G. Dresselhaus and M. S. Dresselhaus. "Formation of general fullerenes by their projection on a honeycomb lattice", Phys. Rev. B, 45, 13834-13836, 1992.

[5] R. Saito, M. Fujita, G. Dresselhaus and M. S. Dresselhaus, "Electronic structure of graphene tubules based on C60", Phys. Rev. B 46, 1804, 1992.

[6] C. J. Cramer, "Essentials of Computational Chemistry", John Wiley \& Sons, Inc., USA, 2002.

[7] C. M. Goringe, D. R. Bowler, and E. Hernández, "Tight-binding modelling of materials", Rep. Prog. Phys. 60, 1447, 1997.

[8] J. W. Mintmire and C. T. White, "Electronic and structure properties of carbon nanotubes", Carbon vol. 33 no 7, 893-902, 1995.

[9] J. W. G. Wildoer, L. C. Venema, A. G. Rinzer, R. E. Smalley and R. E. Smalley and C. Dekker, "Electronic structure of atomically resolved carbon nanotubes", Nature, Vol. 391, p. 59, 1998.

[10]C. T. White, D. H. Robertson and J. W. Mintmire, "Helical and rotational symmetries of nanoscale graphitic tubules", Phys. Rev. B 47 5485-5488, 1993.

[11]C. T. White and J. W. Mintmire, "Fundamental Properties of Single-Wall Carbon Nanotubes", J. Phys. Chem. B 109, 52-65, 2005.

\section{Author Profile}

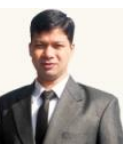

Dr. Rashid Nizam is presently working as an Assistant Professor in Department of Physics, I.F.T.M. University Moradabad. He had received his B.Sc. from H.N.B. Garhwal, Central University in 2004. He had received his M.Sc degree in Physics with specialization in Electronic from H.N.B. Garhwal, Central University in 2006. In 2007 he did Graphic \& Web Designing diploma from A.C.P.L. He had received Ph.D, Degree in Nanotechnology (Physics) from Aligarh Muslim University, India. He had published more than forty one papers in Nanotechnology. His area of interest is to studies of structure and other properties of Carbon Nanotubes and other Clusters using Various Simulation Techniques. He mainly works on the material science using nanotechnology. He had received many rewards from the various universities in presenting the research papers.

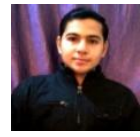

Mirza Mohd Sehban born in 14 December 1992. He had graduated in Physics Honour from Aligarh Muslim University, India. Then he qualified his M.Sc. degree with distinction in Physics with specialization in Electronic from I.F.T.M. University Moradabad in 2015. He is presently pursing B. Ed. from Rashida Begum Muslim Degree College, Amroha. He had published five international papers. Further he is interested in doing research (simulation of different molecules) such that to find "the properties of carbon and carbon derivatives". 


\section{International Journal of Science and Research (IJSR) \\ ISSN (Online): 2319-7064}

Index Copernicus Value (2015): 78.96 | Impact Factor (2015): 6.391

Table 1: Comparison of the band gaps of different PCNTs with increase in diameters

\begin{tabular}{|c|c|c|c|c|c|c|c|c|}
\hline 1. & 2. & 3. & 1. & 2. & 3. & 1. & 2. & 3. \\
\hline$\theta=0^{\circ}$ & & & $\theta=1.87^{\circ}$ & & & $\theta=7.58^{\circ}$ & & \\
\hline$(n, m)$ & Diameter (nm) & Gap (eV) & $(n, m)$ & Diameter (nm) & Gap $(e V)$ & $(n, m)$ & Diameter (nm) & Gap (eV) \\
\hline$(4,0)$ & \begin{tabular}{|l|}
0.31315 \\
\end{tabular} & 2.4853 & $(6,2)$ & 0.56455 & 1.453 & $(6,1)$ & \begin{tabular}{|l|}
0.51337 \\
\end{tabular} & 1.72 \\
\hline$(5,0)$ & 0.39144 & 2.2918 & $(12,4)$ & 1.1291 & 0.765 & $(12,2)$ & 1.0267 & 0.811 \\
\hline$(7,0)$ & 0.54802 & 1.4819 & $(15,5)$ & 1.4114 & 0.596 & $(24,4)$ & 2.0535 & 0.419 \\
\hline$(8,0)$ & 0.62631 & 1.4078 & $(21,7)$ & 1.9759 & 0.435 & $(30,5)$ & 2.5669 & 0.329 \\
\hline$(10,0)$ & 0.78289 & 1.0534 & $(24,8)$ & 2.2582 & 0.374 & $(42,7)$ & 3.5936 & 0.239 \\
\hline$(11,0)$ & 0.86118 & 1.015 & $(30,10)$ & 2.8227 & 0.304 & - & - & - \\
\hline$(13,0)$ & 1.0178 & 0.8168 & $(33,11)$ & 3.105 & 0.273 & - & - & - \\
\hline$(14,0)$ & 1.096 & 0.7934 & $(39,13)$ & 3.6696 & 0.233 & - & - & - \\
\hline$(16,0)$ & 1.2526 & 0.6668 & - & - & - & - & - & - \\
\hline$(17,0)$ & 1.3309 & 0.6511 & $\theta=5.20^{\circ}$ & & & $\theta=8.94^{\circ}$ & & \\
\hline$(19,0)$ & 1.4875 & 0.5634 & $(n, m)$ & Diameter (nm) & Gap & $(n, m)$ & Diameter (nm) & Gap \\
\hline$(20,0)$ & 1.5658 & 0.5521 & $(9,1)$ & \begin{tabular}{|l|}
0.74683 \\
\end{tabular} & 1.1729 & $(5,1)$ & \begin{tabular}{|l|}
0.43589 \\
\end{tabular} & 1.845 \\
\hline$(22,0)$ & 1.7224 & 0.4877 & $(18,2)$ & 1.4937 & 0.5614 & $(10,2)$ & 0.87179 & 0.999 \\
\hline$(23,0)$ & 1.8006 & 0.4792 & $(36,4)$ & 2.9873 & 0.2873 & $(20,4)$ & 1.7436 & 0.482 \\
\hline$(25,0)$ & 1.9572 & 0.4299 & $(45,5)$ & 3.7341 & 0.2268 & $(25,5)$ & 2.1795 & 0.395 \\
\hline$(28,0)$ & 2.1921 & 0.3844 & - & - & - & $(35,7)$ & 3.0513 & 0.277 \\
\hline$(31,0)$ & 2.4269 & 0.3476 & $\theta=5.81^{\circ}$ & & & $(40,8)$ & 3.4871 & 0.246 \\
\hline$(32,0)$ & 2.5052 & 0.3432 & $(n, m)$ & Diameter (nm) & Gap & - & - & - \\
\hline$(34,0)$ & 2.6618 & 0.3172 & $(8,1)$ & 0.6689 & 1.227 & - & - & - \\
\hline$(35,0)$ & 2.7401 & 0.3136 & $(16,2)$ & 1.3378 & 0.647 & - & - & - \\
\hline$(37,0)$ & 2.8967 & 0.2917 & $(32,4)$ & 2.6756 & 0.316 & - & - & - \\
\hline$(38,0)$ & 2.975 & 0.2886 & $(40,5)$ & 3.3445 & 0.256 & - & - & - \\
\hline$(40,0)$ & 3.1315 & 0.27 & - & - & - & - & - & - \\
\hline
\end{tabular}

Table 1: Continued

\begin{tabular}{|c|c|c|c|c|c|}
\hline 1. & 2. & 3. & 1. & 2. & 3. \\
\hline$\theta=19.10^{\circ}$ & & & $\theta=23.41^{\circ}$ & & \\
\hline$(n, m)$ & Diameter (nm) & Gap (eV) & $(n, m)$ & Diameter (nm) & Gap \\
\hline$(4,2)$ & 0.4143 & 2.084 & $(3,2)$ & 0.3413 & 2.377 \\
\hline$(8,4)$ & 0.8285 & 1.009 & $(6,4)$ & 0.6825 & 1.254 \\
\hline$(10,5)$ & 1.0357 & 0.831 & $(12,8)$ & 1.365 & 0.62 \\
\hline$(14,7)$ & 1.4499 & 0.582 & $(15,10)$ & 1.7063 & 0.501 \\
\hline$(22,11)$ & 2.2785 & 0.376 & $(21,14)$ & 2.3888 & 0.355 \\
\hline$(26,13)$ & 2.6927 & 0.315 & $(27,16)$ & 2.947 & 0.29 \\
\hline$(28,14)$ & 2.8999 & 0.295 & $(33,20)$ & 3.6293 & 0.234 \\
\hline$(32,16)$ & 3.3141 & 0.256 & - & - & - \\
\hline$(34,17)$ & 3.5213 & 0.243 & - & - & - \\
\hline$(38,19)$ & 3.9355 & 0.216 & - & - & - \\
\hline- & - & - & - & - & - \\
\hline
\end{tabular}

Volume 6 Issue 1, January 2017 www.ijsr.net 